PHYSICAL REVIEW B 73, 155404 (2006)

\title{
Spectroscopy and nonlinear microscopy of Au nanoparticle arrays: Experiment and theory
}

\author{
Andreas Hohenau and Joachim R. Krenn \\ Karl-Franzens University and Erwin Schrödinger Institute for Nanoscale Research, A-8010 Graz, Austria \\ Jonas Beermann and Sergey I. Bozhevolnyi \\ Department of Physics and Nanotechnology, Aalborg University, DK-9220 Aalborg Øst, Denmark
}

Sergio G. Rodrigo and Luis Martin-Moreno

Facultad de Ciencias, Universidad de Zaragoza, E-50009 Zaragoza, Spain

Francisco Garcia-Vidal

Universidad Autonoma de Madrid, Madrid, Spain

(Received 3 January 2006; published 5 April 2006)

\begin{abstract}
Regular arrays of rectangular gold nanoparticles on glass substrates are characterized by using linear extinction spectroscopy (in the wavelength range of 450-950 nm) and nonlinear scanning optical microscopy, in which two-photon photoluminescence (TPL) excited with a strongly focused laser beam (in the wavelength range of $720-800 \mathrm{~nm})$ is detected. The dimensions of the nanoparticles $\left(\sim 150 \times 150 \times 50 \mathrm{~nm}^{3}\right)$ are chosen to realize the localized-surface-plasmon (LSP) resonance at the wavelength of $\sim 750 \mathrm{~nm}$, which is clearly seen on the obtained extinction spectra as well as with the recorded TPL images. Extinction spectra are modeled using a finite-difference time-domain approach with the dielectric function of gold approximated by a Drude-Lorentz formula, showing rather good agreement between the experimental and theoretical spectra simulated for the nominal geometrical parameters of gold nanoparticles. The developed modeling tool is further used to evaluate the field intensity enhancement at the particles, which is then compared to the intensity enhancement estimated from the TPL images. We find good agreement between the intensity enhancement levels and indications that the LSP resonance wavelengths seen in the extinction spectra might differ from those deduced from the intensity enhancement spectra. The implications of the results obtained are discussed.
\end{abstract}

DOI: 10.1103/PhysRevB.73.155404

PACS number(s): 78.67.Bf, 42.65.-k, 73.20.Mf, 02.70.Bf

\section{INTRODUCTION}

Sub-wavelength-sized metal particles can exhibit optical resonances associated with resonant collective electron oscillations known as localized surface plasmons (LSPs). ${ }^{1}$ Excitation of LSPs results in the occurrence of pronounced extinction bands and in local field enhancement effects. The latter effects are of high interest, in general, for basic physics and electrodynamics, ${ }^{2}$ and of extreme importance for various applied research areas, such as surface enhanced Raman spectroscopy ${ }^{3}$ and microscopy including optical characterization of individual molecules. ${ }^{4}$

The search for configurations ensuring reliable realization of strongly enhanced local fields was often conducted with the help of linear extinction spectroscopy, where minima in the transmitted light intensity were associated with the LSP excitation (see Ref. 5 and references therein). This characterization technique is considered reliable but lacking spatial resolution, since the extinction spectra are influenced by a whole illuminated area containing many nanoparticles. In addition, the information obtained is not direct, since the extinction is determined by the absorption and scattering of individual particles as well as by the scattering diagram of a given particle array. ${ }^{6}$ A more direct approach for the evaluation of local field intensity enhancement has been recently developed utilizing two-photon-excited luminescence in gold. ${ }^{7}$ This technique is based on the circumstance that strongly enhanced local fields due to the excitation of LSPs in gold nanostructures give rise to two-photon absorption, which in turn leads to a broad emission continuum (generated by interband transitions of $d$-band electrons into the conduction band) known as two-photon-excited (photo)luminescence (TPL) in gold. ${ }^{8}$ It has been demonstrated that nonlinear scanning optical microscopy, in which the TPL excited with a strongly focused laser beam is detected, can be used for characterization of the local field intensity enhancement in gold nanoparticles (bow-tie nanoantennas) and at their surfaces. ${ }^{7}$ However, it was difficult to ascertain the accuracy of the main formula used in the developed approach, since a crucial parameter, viz., the area of TPL origin, had to be found in the course of simulations. ${ }^{7}$ It would be advantageous to verify this approach by using the same modeling tool for the (same) scattering system investigated not only with the TPL but also with another, preferably wellestablished, experimental technique (e.g., extinction spectroscopy).

In this work, we combine the results of TPL microscopy (in the wavelength range of 720-800 nm) with theoretical modeling based on the finite-difference time-domain method, where only the independently determined geometry and dielectric functions of the sample were used as input without any free fitting parameters. Additionally, the theoretical results are compared carefully to experimentally recorded extinction spectra (in the wavelength range of 450-950 nm) to verify the quality of the modeling. As samples we use regular arrays of rectangular gold nanoparticles on glass substrates 
for which we recently obtained strong, wavelengthdependent TPL signals. ${ }^{9}$ Similar systems have been previously investigated with the extinction spectroscopy in the visible and near-infrared spectral range. ${ }^{5}$ In general, the individual LSP resonances of nanoparticles can be tuned by varying both particle dimensions and shapes, ${ }^{10}$ while the resonances stemming from multiple interactions within the grating of particles (scatterers) can be tuned by varying the grating period or gap between particles. ${ }^{5,7,11}$ We pay special attention to the case when the individual LSP resonance is matched to the grating resonance since one could expect a strong modification of the field intensity enhancement compared to the "normal" 2,7 case.

The paper is organized as follows. In Sec. II the investigated arrays and the characterization techniques are outlined followed by a brief description of the developed modeling approach. Section III presents the results obtained and their discussion, starting with the comparison of the measured and simulated extinction spectra for different array periods and sizes of nanoparticles. The ensuing discussion is supported with the field intensity distributions calculated at different cross sections of an individual particle. The results of TPL microscopy are then presented and used to evaluate the field intensity enhancement ${ }^{7,9}$ for different wavelengths and compare them with the simulated values. We offer our conclusions in Sec. IV which terminates the paper.

\section{SAMPLE DESCRIPTION AND METHODS}

\section{A. Experiment}

The samples consist of two-dimensional arrays of nominally rectangular gold particles on top of a 0.5 -mm-thick glass substrate (Fig. 1) produced by electron beam lithography. ${ }^{12}$ The dimensions of the particles and the grating constants of the arrays are varied to systematically study their influence on the optical extinction and reflection spectra and TPL images. The dimensions of the nanoparticles $\left(\sim 150 \times 150 \times 50 \mathrm{~nm}^{3}\right)$ were chosen to realize the LSP resonance with a polarization parallel to the substrate close to the wavelength of $750 \mathrm{~nm}$, so that its influence could be observed in both optical spectra and TPL images.

Extinction spectra are recorded by a Zeiss MMS-1 microspectrometer attached to a conventional optical microscope [see Fig. 2(a)] equipped with a $10 \times, 0.25$ numerical aperture objective. To control the polarization of the incident light, a polarizer is inserted in the optical path of the microscope. The extinction is calculated from the transmission of the arrays on the substrate $(T)$ with reference to the transmission of the bare substrate $\left(T_{\text {glass }}\right)$ as $\log _{10}\left(T_{\text {glass }} / T\right)$.

The experimental setup for TPL scanning microscopy is schematically shown in Fig. 2(b) and described in detail elsewhere. ${ }^{9}$ It allows us to simultaneously record the TPL signal (detected by a photomultiplier) and the backscattered light (detected by a photodiode) as a function of the sample position with the resolution determined by a focal spot size of the excitation laser at the sample surface $(\sim 1 \mu \mathrm{m}) .{ }^{13} \mathrm{In}$ these experiments, we use a $200 \mathrm{fs}$ Ti:sapphire laser at a repetition rate of $80 \mathrm{MHz}$ with a linewidth of $\sim 10 \mathrm{~nm}$ and (a)
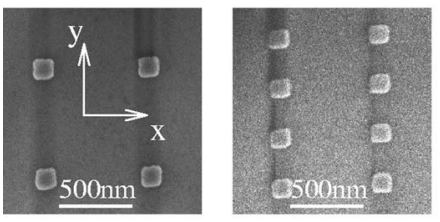

(b)

(c)

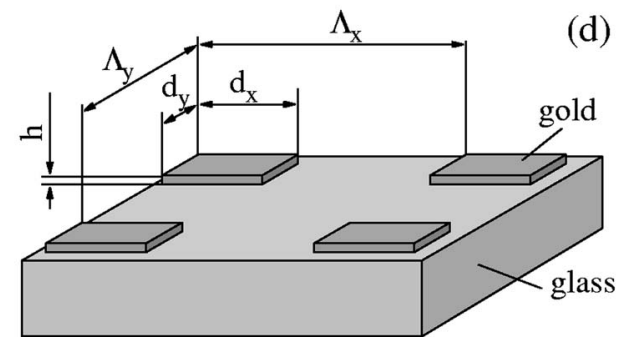

FIG. 1. Scanning electron microscopy images of the investigated arrays of rectangular gold nanoparticles on glass substrate with (a) $\Lambda_{x}=\Lambda_{y}=740 \mathrm{~nm}$ and $d_{x}=d_{y}=130 \mathrm{~nm}$ (type A), (b) $\Lambda_{x}$ $=2 \Lambda_{y}=740 \mathrm{~nm}$ and $d_{x}=d_{y}=130 \mathrm{~nm}$ (type B), and (c) $\Lambda_{x}=\Lambda_{y}$ $=740 \mathrm{~nm}$ and $d_{x}=0.5 d_{y}=130 \mathrm{~nm}$ (type C). (d) Sketch of the arrays defining the different parameters. The array and particle dimensions were varied between the different samples in the ranges of (all dimensions in $\mathrm{nm}) 130 \leqslant d_{x} \leqslant 160,65 \leqslant d_{y} \leqslant 320, h=25$ or 50 , $740 \leqslant \Lambda_{x} \leqslant 860$, and $370 \leqslant \Lambda_{y} \leqslant 1740$.

an adjustable polarization plane. The laser wavelength can be tuned between 720 and $900 \mathrm{~nm}$, which allows us to make spectrally resolved studies of TPL efficiency. The typical average incident power is in the range of 0.1 to $50 \mathrm{~mW}$. Compared to the previous measurements ${ }^{9}$ the setup has now been improved so that the average dark counts are reduced by one order of magnitude to only $\sim 20$ counts per second.

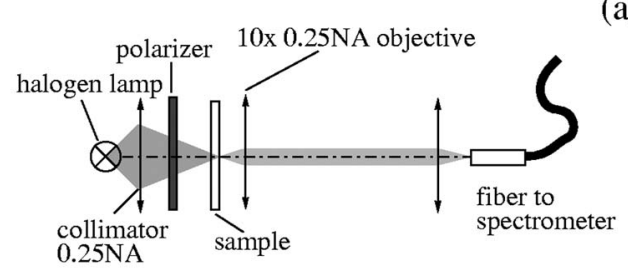

(a)

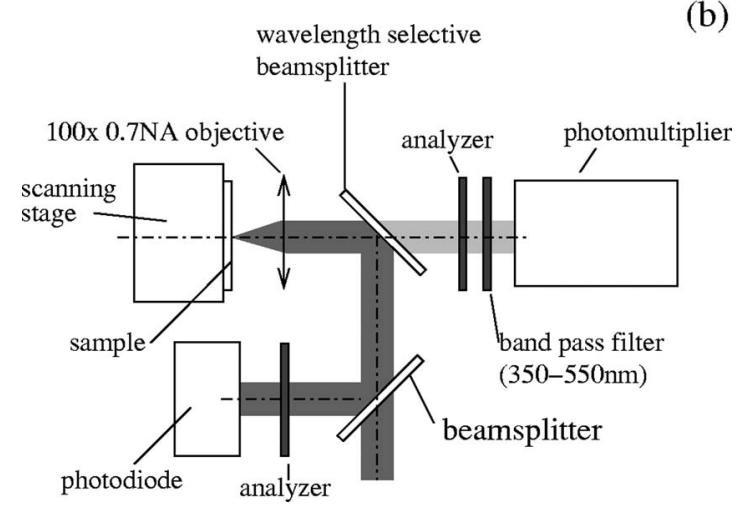

FIG. 2. (a) Microspectrometer setup for extinction measurements and (b) experimental setup for TPL microscopy. 


\section{B. Simulations}

For finite-difference time-domain (FDTD) simulations we modeled the sample as gold particles on top of a semiinfinitely extending glass substrate. The fields were calculated in a box of $\Lambda_{x} \times \Lambda_{y} \times 1.3 \mu \mathrm{m}^{3}$ with periodic boundary conditions at the walls perpendicular to the substrate and a "uniaxial perfect matched layer"14 at the walls parallel to the substrate. Typically the cell size was $5 \times 5 \times 5 \mathrm{~nm}^{3}$ in space and the time steps were $0.0077 \mathrm{fs}$ (corresponding to a Courant-Friedrich-Levy factor of 0.8 ), to guarantee the numerical stability of the three-dimensional simulations. Moreover, we found that after a simulation time of $100 \mathrm{fs}$, convergency was guaranteed. The dielectric function for gold was approximated by the Drude-Lorentz formula $\epsilon(\omega)=\epsilon_{r}$ $-\omega_{P}^{2} / \omega /(\omega+l \gamma)-\Delta \epsilon \Omega^{2} /\left[\left(\omega^{2}-\Omega^{2}\right)+\imath \Gamma \omega\right]$, with $\epsilon_{r}=5.9673$, $\omega_{p}=8.7292 \mathrm{eV}, \gamma=0.0650 \mathrm{eV}, \Delta \epsilon=1.09, \Omega=2.6848 \mathrm{eV}$, and $\Gamma=0.43307 \mathrm{eV} .^{15}$ This functional form for $\epsilon(\omega)$ allows the use of the piecewise linear recursive convolution method for the FDTD simulations of metals. ${ }^{16,17}$ In the simulations, the structures were excited by a Gaussian wave packet composed of plane waves with wave vector $\mathbf{k}$ perpendicular to the substrate and all frequencies of interest. Spectra were calculated after projection onto diffracted modes. In the comparison with experimental data, only the zero-order mode was considered in the postprocessing, as experimental intensities were collected in a small solid angle centered around the normal direction.

\section{RESULTS AND DISCUSSION}

We first investigate extinction spectra of the fabricated arrays of gold particles on glass substrate and compare them to the results of numerical simulations, aiming at identification of the LSP resonances and understanding of their interrelations with the particle shapes and sizes as well as the array periods in analogy to the previous studies of arrays of ellipsoidal particles. ${ }^{1,6}$

\section{A. Spectroscopy}

To demonstrate the influence of particle shape and array parameters on the extinction spectra, we focus in the following on three different types of arrays (see Fig. 1): (A) arrays with $\Lambda_{x}=\Lambda_{y}$ and $d_{x}=d_{y}$ (quadratic array of quadratic particles), (B) arrays with $\Lambda_{x}=2 \Lambda_{y}$ and $d_{x}=d_{y}$ (rectangular array of quadratic particles), and (C) arrays with $\Lambda_{x}=\Lambda_{y}$ and $2 d_{x}$ $=d_{y}$ (quadratic array of rectangular particles). For all arrays, we kept $\Lambda_{x}=740 \mathrm{~nm}$ and recorded spectra for $d_{x}=130,140$, 150 , and $160 \mathrm{~nm}$.

Figure 3 depicts the experimental and simulated extinction spectra of arrays of type A. The dominating feature in the experimental spectra is one broad extinction peak with a maximum between 710 and $810 \mathrm{~nm}$, depending on the polarization and particle size. In analogy to ellipsoidal particles, this extinction peak can be attributed to the excitation of the LSP resonance with dominating dipolar character. Since the particles are rectangular in shape, we can assume that several excited LSP eigenmodes contribute to this extinction peak. ${ }^{1}$ With increasing lateral particle dimensions (the particle
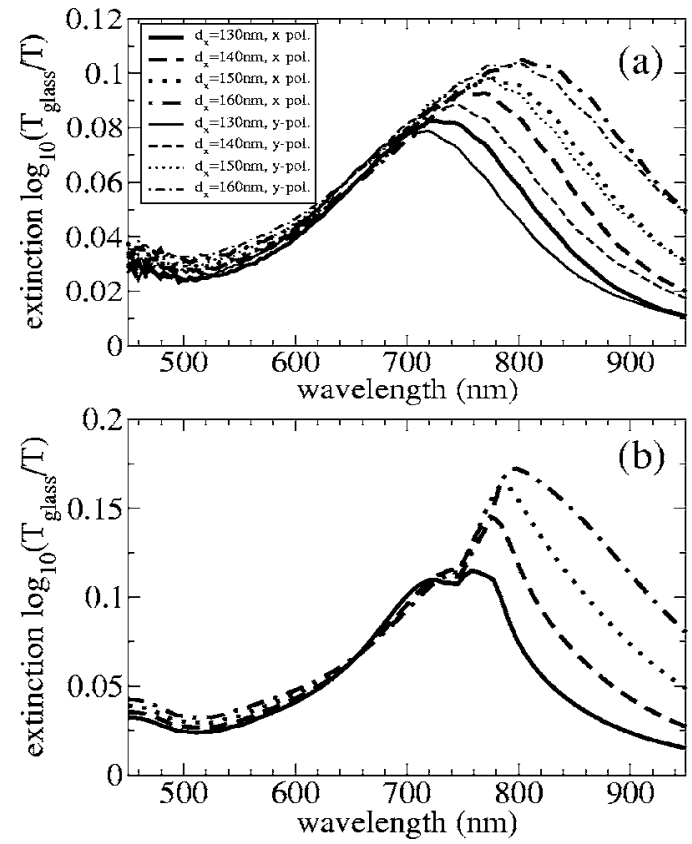

FIG. 3. Experimental (a) and simulated (b) extinction spectra of type-A arrays with $\Lambda_{x}=\Lambda_{y}=740 \mathrm{~nm}$ and $d_{x}=d_{y}=130,140,150$, and $160 \mathrm{~nm}$

height was kept constant) we observe a redshift and an increase in extinction strength, as would be the case for arrays of simple ellipsoidal particles of increasing size as well. The slight blueshift of the spectra recorded with polarization parallel to the $y$ axis compared to those recorded with polarization parallel to the $x$ axis stems from a slight asymmetry in the actual $x$ and $y$ dimensions of the particles.

The simulated spectra [Fig. 3(b)] agree very well, both quantitatively and qualitatively, with the experimental spectra, though exhibiting small (but distinct) additional dips at 740 and $550 \mathrm{~nm}$. These dips coincide with the excitation of the first grating orders propagating (nearly) parallel to the sample interface on the air and substrate sides of the particles, respectively. We attribute the fact that these dips are not very pronounced in the experimental spectra to experimental conditions of spectra recording (integration over a certain solid angle due to the finite numerical aperture of the microspectrometer) as well as to the influence of finite array size and imperfections of the sample.

In the case of type-A arrays, due to the large grating constant, optical near-field interactions between the particles only weakly influence the spectral position of the LSP resonances. ${ }^{18}$ This was verified by comparing the recorded extinction spectra of type-A arrays with grating constants ranging from 740 to $860 \mathrm{~nm}$ (not shown). Nevertheless, a considerable effect of the grating constant is observed for arrays of type $\mathrm{B}$, whose extinction spectra exhibit not only the expected redshift for increasing particle sizes but also a remarkable difference between the spectra obtained for $x$ and $y$ polarizations (see Fig. 4). Such a strong effect can be explained as follows.

For type-B arrays, the grating constant parallel to the $y$ axis, $\Lambda_{y}$, is only $370 \mathrm{~nm}$, i.e., the array periodicity is lower than the wavelength of light in air for (vacuum) wavelengths 

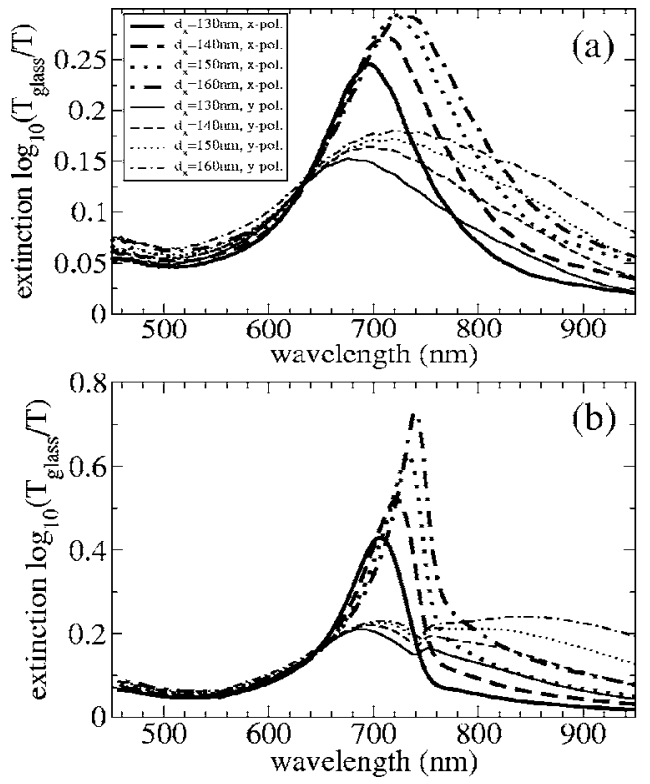

FIG. 4. Experimental (a) and simulated (b) extinction spectra of type-B arrays with $\Lambda_{x}=2 \Lambda_{y}=740 \mathrm{~nm}$ and $d_{x}=d_{y}=130,140,150$, and $160 \mathrm{~nm}$.

larger than $370 \mathrm{~nm}$ and of light in the substrate (refractive index $n=1.52$ ) for (vacuum) wavelengths larger than $550 \mathrm{~nm}$. Therefore, the coherently excited (dipolar) LSPs can emit in the $y-z$ plane only to the zeroth grating order. In addition, for the polarization parallel to the $x$ axis, diffraction into the first grating order in the $x-z$ plane is weak due to the dipolar far-field emission characteristic. Therefore, the radiation damping for $x$ polarization is considerably reduced, resulting in narrower widths and stronger maxima observed for the corresponding extinction peaks (compare Figs. 3 and 4). At the same time, for $y$ polarization, the field scattered by one particle is of comparable strength and approximately in counterphase (due to retardation and the phase shift between LSP and exciting field) with the incident field at the position of its next neighbors in the $y$ direction, thereby decreasing the total field experienced by the particles. This leads to a decrease of the extinction (normalized to the particle density) compared to arrays of type A.

Also in the case of type-B arrays we find good agreement of the simulated extinction spectra [Fig. 4(b)] with the experimental ones, except for the behavior observed at $740 \mathrm{~nm}$, where the simulations show strong dips or peaks related to the occurrence of a grazing grating order on the air side. For $y$ polarization, we find a similar dip in the spectra as for type-A arrays, but for $x$ polarization we observe a sharp peak when the extinction peak of the particles coincides with the grating constant ( $d=160 \mathrm{~nm}$; thick dash-dotted curve).

This behavior can be qualitatively accounted for by the aforementioned effect of coherent superposition of the LSP fields (driven by the incident field) with the fields scattered by the neighboring particles. For the array with the particle size $d=160 \mathrm{~nm}$, the particle separations in terms of light wavelength $\lambda$ at the LSP extinction peak are $\Lambda_{y}=\lambda / 2$ and $\Lambda_{x}=\lambda$, respectively. Assuming the LSP scattered fields to be qualitatively similar to the fields emitted by a point dipole,

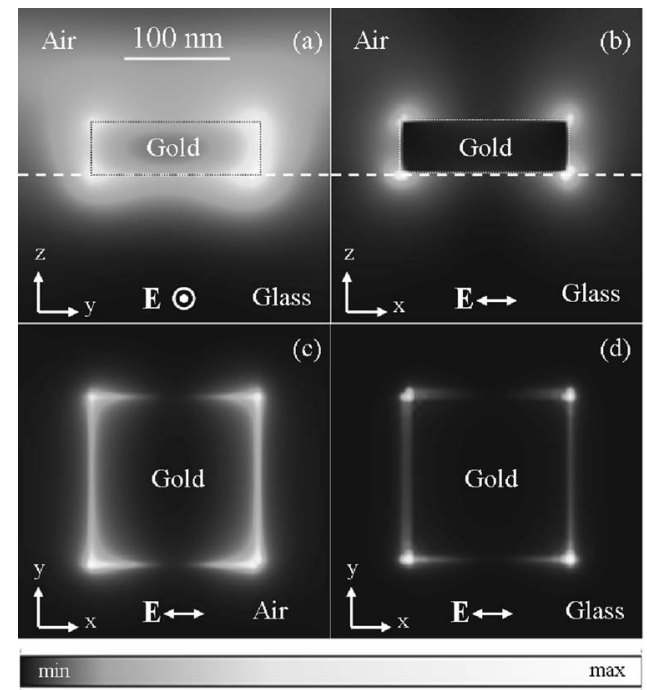

FIG. 5. Simulated distribution of the local field intensity enhancement $\left|E_{\text {bump }}(\mathbf{r}, \lambda)\right|^{2} /\left|E_{\text {film }}(\lambda)\right|^{2}$ around a single gold particle $\left(d_{x}=d_{y}=160 \mathrm{~nm}\right.$, height $\left.d_{z}=50 \mathrm{~nm}\right)$ positioned on a glass substrate in a type-B lattice with $\Lambda_{x}=2 \Lambda_{y}=740 \mathrm{~nm}$ and obtained for an $x$-polarized (resonant case) exciting electric field $E$ as indicated on each image. The distributions are taken in planes either through the center of the particle (a),(b) or from the top (c) and bottom (d) surface of the particle as indicated by coordinate axes on each image. The maximum levels are (a) $\sim 102$, (b) $\sim 820$, (c) $\sim 2300$, and (d) $\sim 20000$.

the electric field in the direction of the induced LSP is dominating over that in the transverse direction (in this distance regime). Due to retardation the scattered field of one particle at the position of its next neighbor (in the direction of the dipole) has a phase shift close to $\pi$ for $y$ polarization or $2 \pi$ for $x$ polarization, respectively. Therefore either destructive or constructive interference occurs, which is responsible for the dip or peak in the extinction spectrum. This effect is clearly seen in the distributions of optical near-field intensity calculated for different polarizations. For $x$ polarization (Fig. $5)$, there is constructive interference of the LSP fields of one particle and the fields scattered by its neighboring particles, similar to the resonance of a series resonant circuit. Therefore the field intensities at the top corners of the particle are very strong. In contrast, for $y$ polarization (Fig. 6), we find (due to destructive interference) very weak field intensities at the top corners of the particle, similar to the antiresonance of a parallel resonant circuit. This spectrally sharp constructive or destructive interference of the calculated local fields also changes the field strength within the particles and is therefore recognized in the far field by a decrease or increase of the extinction. However, this effect is not readily observed in the experimentally recorded spectra, which we attribute to local phase variations of the LSP oscillations due to slight size variations (inhomogeneous broadening), particle distance variations, the finite size of the experimentally investigated arrays, and the finite numerical aperture of our spectrometer, i.e., a distribution of $\mathbf{k}$ vectors in excitation and detection.

Finally, let us consider the influence of the particle shape on the spectra. Figure 7 displays the experimental and simulated extinction spectra for type-C arrays with $\Lambda_{x}=\Lambda_{y}$ 


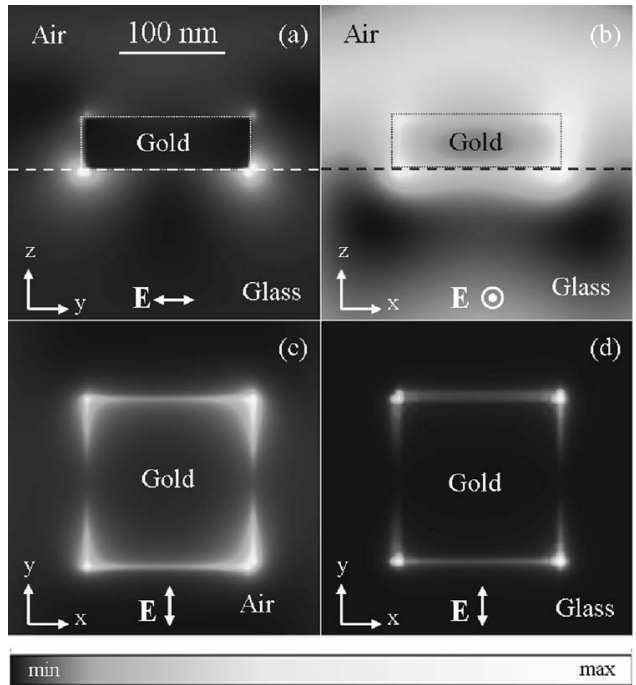

FIG. 6. The same as Fig. 5, but obtained for a $y$-polarized (antiresonant case) exciting electric field $E$ as indicated on each image. The maximum levels are (a) $\sim 138$, (b) $\sim 13$, (c) $\sim 157$, and (d) $\sim 3100$.

$=740 \mathrm{~nm}$ and rectangular particles with $d_{y}=2 d_{x}$. Whereas for $x$ polarization the extinction looks similar to that of type-A arrays but with somewhat broader peaks, for $y$ polarization (parallel to the largest particle dimension) the extinction is very low, featuring a weakly pronounced peak (in both experimental and simulated spectra) close to $570 \mathrm{~nm}$, which we attribute to multipolar LSP excitation. Due to the changed particle geometry with doubled $y$ dimension, the dipolar LSP
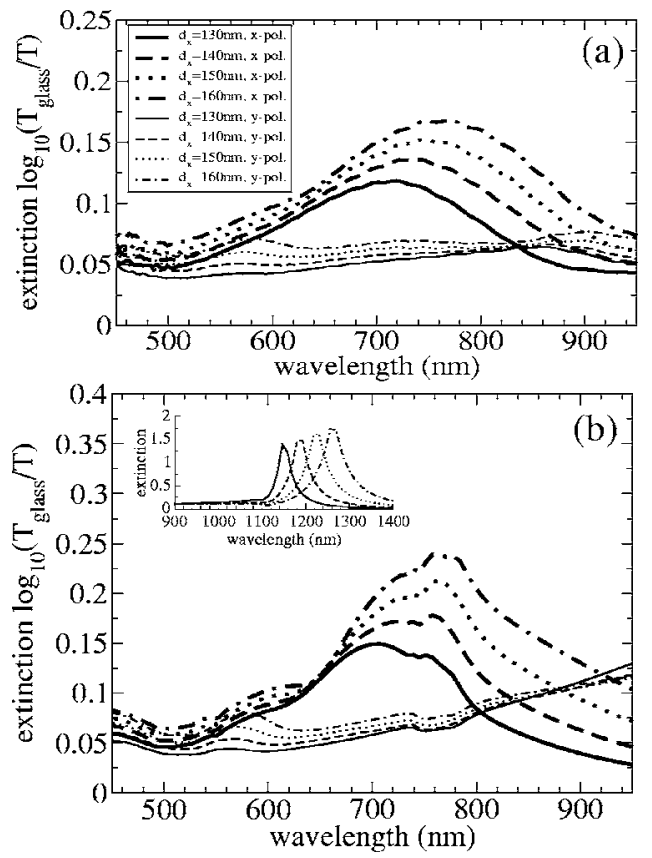

FIG. 7. Experimental (a) and simulated (b) extinction spectra of type-C arrays with $\Lambda_{x}=\Lambda_{y}=740 \mathrm{~nm}$ and $d_{x}=0.5 d_{y}=130,140,150$, and $160 \mathrm{~nm}$. The inset in (b) depicts the simulated extinction spectra for $d_{x}=160 \mathrm{~nm}$ and $y$ polarization in the spectral range from 900 to $1400 \mathrm{~nm}$.
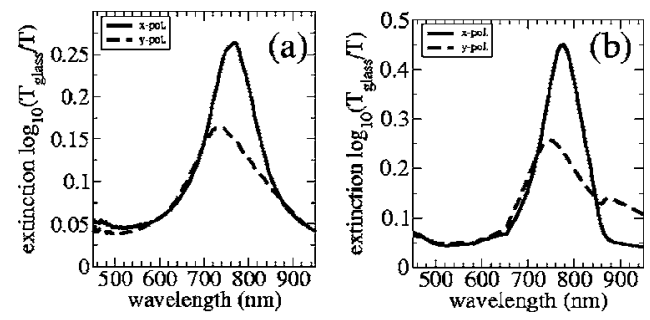

FIG. 8. Experimental (a) and simulated (b) extinction spectra of a gold nanoparticle array with $\Lambda_{x}=860 \mathrm{~nm}, \Lambda_{y}=430 \mathrm{~nm}, d_{x}=d_{y}$ $=160 \mathrm{~nm}$.

peak (that can be excited with $y$ polarization) is shifted to near-infrared wavelengths (at $\sim 1200 \mathrm{~nm}$ ) as revealed by simulations in a spectrally extended region [see inset in Fig. $7(b)]$.

\section{B. TPL microscopy}

The good agreement found between the simulated and experimental extinction spectra encouraged us to apply the developed modeling tool for simulations of the field intensity enhancement and subsequent comparison with the results obtained with the TPL microscopy. For a first comparison, we decided to choose a sample exhibiting the most pronounced effects, i.e., large extinction values and strong polarization influence. Within the range of geometrical parameters studied in this work, we found the sample having square 160 $\times 160 \mathrm{~nm}^{2}$ nanoparticles but different grating constants $\Lambda_{x}$ $=860 \mathrm{~nm}$ and $\Lambda_{y}=430 \mathrm{~nm}$ satisfying the premises best. The experimentally measured extinction spectra as well as the results of FDTD simulations for this array are shown in Fig. 8.

The TPL images were recorded at different wavelengths $(730,745,760,775$, and $800 \mathrm{~nm})$ of the fundamental harmonic (FH) illuminating the sample along with the FH reflection images. Figure 9 displays exemplarily the result for the FH wavelength of $760 \mathrm{~nm}$ obtained for two polarization configurations of incident $\mathrm{FH}$ and detected TPL radiation indicated by arrows on the images [the sample orientation corresponds to that shown in Fig. 1(b)]. The image size is $15 \times 15 \mu \mathrm{m}^{2}$ with $75 \times 75$ points and the incident power was kept at $\sim 3 \mathrm{~mW}$ to avoid sample damage.

The FH and TPL images (Fig. 9) were obtained starting $\sim 3 \mu \mathrm{m}$ outside the array of particles. This relatively long distance turned out to be very important in order to get an accurate signal reference from bare glass. The reflection from the glass substrate is rather weak compared to the reflection from gold particles and appears dark in the images. The individual bumps are only resolvable along the $x$ axis where the separation between them is $\sim 700 \mathrm{~nm}$. This is expected as the resolution in FH and TPL images has previously been determined to approximately 1 and $0.7 \mu \mathrm{m}$, respectively. ${ }^{13}$ Overall the $\mathrm{FH}$ images appear similar for the investigated polarization directions and wavelengths, while the TPL images are more different. For $x$-polarized excitation and detection the average TPL signal from the gold particles is relatively high and homogeneous, while the $y$ polarization produces lower average TPL signal but with a few bright 


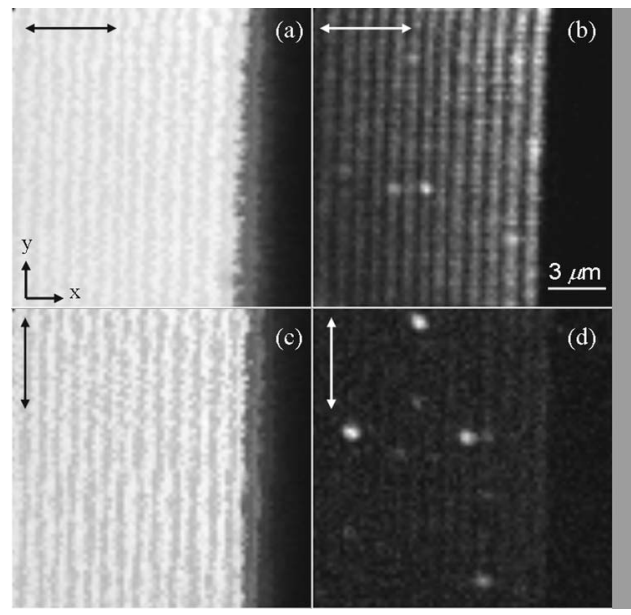

FIG. 9. FH (a),(c) and TPL (b),(d) images of a gold particle array on glass with $\Lambda_{x}=860 \mathrm{~nm}, \Lambda_{y}=430 \mathrm{~nm}$, and $d_{x}=d_{y}=160 \mathrm{~nm}$ obtained using $3 \mathrm{~mW}$ of incident power at the wavelength of $760 \mathrm{~nm}$ for the polarizations indicated by arrows on the images. The maximum TPL signal is (b) $\sim 6200$ and (d) $\sim 2000$ counts/s.

spots appearing clearer on the dark background. Such a polarization dependence was not noticed in previous TPL investigations of square gold bumps in a square array. ${ }^{9}$ With respect to the TPL dependence on excitation wavelength, the highest signal was observed for $x$ polarization and with a resonance around $745 \mathrm{~nm}$. For $y$ polarization, no pronounced maxima were observed and the signal was considerably weaker (in agreement with the spectroscopy measurements). It should be noted that, in general, the absolute TPL signal levels were found to be very sensitive to the focus adjustment and possible gradual damage of the sample. ${ }^{2}$

Based on the method used previously, ${ }^{7,9}$ the average (over the particle area) intensity enhancement factor $\alpha$ observed in the TPL measurements can be estimated by comparing the TPL signals from gold nanoparticles to those from smooth gold films. The appropriate relation is given by

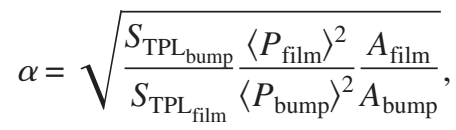

where $S_{\mathrm{TPL}}$ is the obtained TPL signal, $\langle P\rangle$ is the used average incident power, and $A$ is the area generating the TPL signal. Using this relation for the TPL measurements with the $A_{\text {bump }}$ area kept constant, one obtains Fig. 10. At the $745 \mathrm{~nm}$ resonance we find an average intensity enhancement of $\sim 111$ and a maximum (measured at one of the bright spots in Fig. 9) of $\sim 250$ (compared to an average enhancement of 80 and a maximum enhancement of 115 reported previously ${ }^{9}$ ). Considering the double density of particles of our sample compared to the samples of Ref. 9 these higher enhancement values should actually be expected. For our sample configuration $\left(\Lambda_{y}=430 \mathrm{~nm}\right)$ there are rather two than one particle within the focal spot of the exciting laser beam. This larger density of particles can be accounted for in the estimated enhancement by using $A_{\text {bump }}=2 \times(160 \mathrm{~nm})^{2}$, resulting in the average intensity enhancement of $\sim 80$ and a maximum of 177 , values that are quite similar to those for

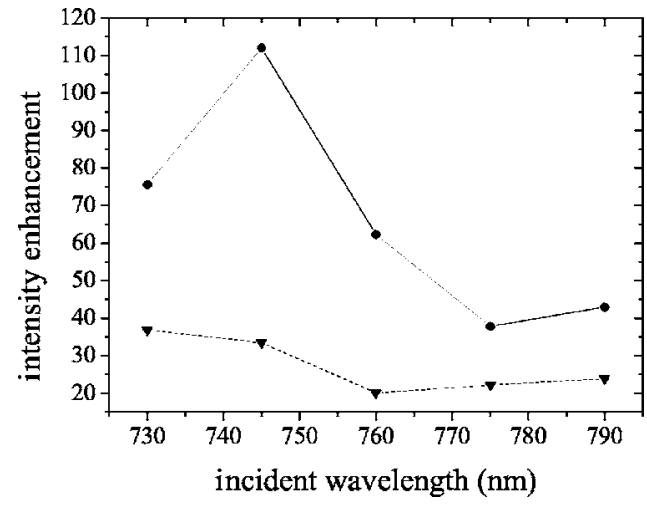

FIG. 10. Spectral dependence of the average TPL enhancement [Eq. (1)] obtained from the particle array. Solid and dashed lines represent $x$ and $y$ polarization, respectively.

the square lattice configuration. ${ }^{9}$ This indicates that the particle near-field interaction of this sample plays only a minor role for the generation of the TPL signal for $x$ polarization.

\section{FDTD results in TPL}

Using simulated intensity enhancement maps, similar to those shown in Figs. 5 and 6 but obtained for several excitation wavelengths, it is possible to estimate the achievable TPL intensity enhancement factor $\alpha(\lambda)$ by evaluating the following expression:

$$
|\alpha|^{2}(\lambda)=\frac{\iint_{A_{\text {bump }}}\left|E\left(x, y, z_{b}, \lambda\right)\right|^{4} d x d y}{\iint_{A_{\text {bump }}}\left|E\left(z_{m}, \lambda\right)\right|^{4} d x d y},
$$

where the quantity $\left|E\left(x, y, z_{b}, \lambda\right)\right|^{4}$ integrated over the top layer of the particle is assumed to be proportional to the TPL signal from the particle (bump) and compared to $\left|E\left(z_{m}, \lambda\right)\right|^{4}$ integrated over the same size of area, but from a smooth gold film without bumps.

In Fig. 11 these intensity enhancement values estimated from the simulations are depicted for the wavelength range 450-1100 nm with $20 \mathrm{~nm}$ steps. As seen in Fig. 11 the simulated TPL intensity enhancement for square particles (160 $\left.\times 160 \mathrm{~nm}^{2}\right)$ in a rectangular lattice geometry $\left(\Lambda_{x}=860 \mathrm{~nm}\right.$,

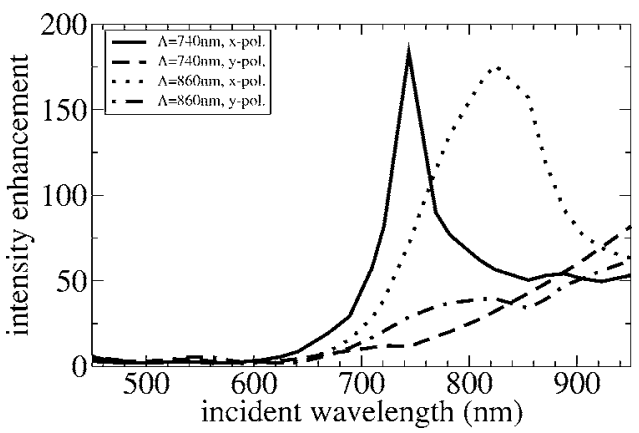

FIG. 11. Simulated spectral dependence of the average TPL enhancement obtained from the particle array. 
$\left.\Lambda_{x}=430 \mathrm{~nm}\right)$ exhibits the same clear polarization dependence as observed in the TPL microscopy measurements. Furthermore, for both simulated and measured intensity enhancements the ratio between $x$ and $y$ polarizations is of the same order of magnitude, though the absolute levels are different. The difference in the peak enhancement levels is most probably related to variation in the sizes of particles in the fabricated sample. It is also seen that in the TPL measurements the peak position is also slightly shifted to shorter wavelengths around $750 \mathrm{~nm}$ (Fig. 10). This shift could be due to the actual particle dimension being somewhat smaller than the nominal particle dimension. The most interesting feature in this context is that, in the simulated field enhancement spectra, one observes a clear redshift of the maximum with the increase of the array period (Fig. 11). We found a similar linear shift also for other array periods (not shown). Note that the extinction spectra depend only weakly on the period in this range. Overall, there are indications that the extinction and field intensity spectra of particle arrays might reach maxima at different wavelengths, but this conjecture requires a separate study that can corroborate its existence and explain its origin.

\section{CONCLUSION}

In conclusion, we combined the results of linear extinction spectroscopy, TPL microscopy, and FDTD simulations to gain insight into the electrodynamical processes involved in the generation of TPL from arrays of rectangular gold nanoparticles. For the simulations we approximated the dielectric function of gold by a Drude-Lorentz formula and found good agreement between measured and calculated spectra with the nominal geometrical parameters of the gold nanoparticles. This demonstrates that FDTD simulations are suitable for describing the electromagnetic behavior of such nanoparticle arrays. The simulations show pronounced effects when the particle resonances spectrally overlap with array resonances. Experimentally we could not observe such effects yet and we attribute this to the geometrical imperfections of the samples and the measurement process. TPL enhancements were found to be in the range of $10^{2}$ with a sharp spectral response. Experimental TPL excitation spectra were well reproduced by FDTD calculations considering $E^{4}$ integrated over the top layer of the particles as the origin of the TPL signal. Additionally, we found indications that the spectral position of the maximum near-field intensity enhancement might differ considerably from the position of the maximum seen in the extinction spectra, depending on the period of the particle array.

\section{ACKNOWLEDGMENTS}

The authors acknowledge support from the European Network of Excellence, Plasmo-Nano-Devices (FP6-2002-IST1-507879) and the STREP "Surface Plasmon Photonics" (FP6-NMP4-CT-2003-505699).
${ }^{1}$ U. Kreibig and M. Vollmer, Optical Properties of Metal Clusters, Springer Series in Materials Science Vol. 25 (Springer, Berlin, 1995).

${ }^{2}$ P. Muhlschlegel, H.-J. Eisler, O. J. F. Martin, B. Hecht, and D. W. Pohl, Science 308, 1607 (2005).

${ }^{3}$ S. Nie and S. R. Emroy, Science 275, 1102 (1997).

${ }^{4}$ J. Azoulay, A. Débarre, A. Richard, and P. Tchénio, J. Microsc. 194, 486 (1999).

${ }^{5}$ N. Félidj, J. Aubard, G. Lévi, J. R. Krenn, G. Schider, A. Leitner, and F. R. Aussenegg, Phys. Rev. B 66, 245407 (2002).

${ }^{6}$ B. Lamprecht, G. Schider, R. T. Lechner, H. Ditlbacher, J. R. Krenn, A. Leitner, and F. R. Aussenegg, Phys. Rev. Lett. 84, 4721 (2000).

${ }^{7}$ P. J. Schuck, D. P. Fromm, A. Sundaramurthy, G. S. Kino, and W. E. Moerner, Phys. Rev. Lett. 94, 017402 (2005).

${ }^{8}$ M. R. Beversluis, A. Bouhelier, and L. Novotny, Phys. Rev. B 68, 115433 (2003).

${ }^{9}$ J. Beermann and S. I. Bozhevolnyi, Phys. Status Solidi C 2, 3983 (2005).

${ }^{10}$ G. T. Boyd, T. Rasing, J. R. R. Leite, and Y. R. Shen, Phys. Rev.

\section{B 30, 519 (1984).}

${ }^{11}$ D. P. Fromm, A. Sundaramurthy, P. J. Schuck, G. S. Kino, and W. E. Moerner, Nano Lett. 4, 957 (2004).

${ }^{12}$ M. A. McCord and M. J. Rooks, Handbook of Microlithography, Micromachining and Microfabrication (SPIE and the Institute of Electrical Engineers, Bellingham, WA, 1997), Vol. 1.

${ }^{13}$ J. Beermann, S. I. Bozhevolnyi, K. Pedersen, and J. FagePedersen, Opt. Commun. 221, 295 (2003).

${ }^{14}$ L. Zhao and A. C. Cangellaris, IEEE Trans. Microwave Theory Tech. 44, 2555 (1996).

${ }^{15}$ A. Vial, A. S. Grimault, D. Macias, D. Barchiesi, and M. L. de la Chapelle, Phys. Rev. B 71, 085416 (2005).

${ }^{16}$ R. J. Luebbers, IEEE Trans. Antennas Propag. 40, 1297 (1992).

${ }^{17}$ A. Taflove and S. C. Hagness, Computational Electrodynamics: The Finite-Difference Time-Domain Method (Artech House, Boston, 2000).

${ }^{18}$ W. Rechberger, A. Hohenau, A. Leitner, J. R. Krenn, B. Lamprecht, and F. R. Aussenegg, Opt. Commun. 220, 137 (2003). 